DOI: $10.12731 / 2070-7568-2021-10-4-143-160$

УДК 334.7.01

ИССЛЕДОВАНИЕ ТЕОРЕТИЧЕСКИХ ПОДХОДОВ

И ПРАКТИК УПРАВЛЕНИЯ ВЗАИМОДЕЙСТВИЕМ

ОСНОВНЫХ СУБЪЕКТОВ, СПОСОБСТВУЮЩИХ

ОБЕСПЕЧЕНИЮ УСТОЙЧИВОГО РАЗВИТИЯ РЕГИОНА

В СТРАТЕГИЧЕСКОЙ ПЕРСПЕКТИВЕ

\author{
Кириллова $E . A$.
}

В сущуествующих концеепиях определения источников конкурентных преимуществ достаточно слабо представлены инструменты анализа и оценки ресурсов, способностей и компетеничий применительно для коопераџионного формирования. Хотя как показывает зарубежная практика именно такие формы совместной организации деятельности в настоящее время имеют наибольшую эффективность. Эти особенности определили иель настоящего исследования - анализ существующих теоретических подходов и зарекомендовавщих себя практик идентификации и оценки уникальных ресурсов и способностей сложных сочиально-экономических систем.

Метод или методология проведения работы: использовались преимущественно общелогические методы анализа и синтеза, обобщзения. Результаты показывают, что одним из источников конкурентных преимуществ современных организаций выступает сеть их партнерств, важную роль в которой играют научные организации. В тоже время они не статичны, а представляют собой постоянно меняюшуюся комбинацию свойств, параметров, навыков, компетенций, интересов и способностей, что определяет потребность в описании таких конкурентных преимуществ через способности «действия» - способность системы развиваться, обучаться, наращцивать объемы ресурсов и повыпать эффективность их использования за счет интегрирования, формирования, рекомбинации и ре- 
конфигурации ключевых компетенций, в интересах соответствия изменяюшейся внешней среды, тем самым обеспечивая стратегическую гибкость организации посредством реализации максимально возможного синергетического эффекта от совокупности имеющиихся ресурсов и компетенций. Возрастает потребность в такой модели реализации инновационной деятельности как «тройная спираль». Такое изменение состава участников приводит к изменению движений информационных, финансовых и материальных потоков внутри системы кооперационного формирования, трансформации ее структуры, целевых установок и распределения ресурсов для их достижения. Роли самих субъектов взаимодействия сейчас также достаточно сильно меняются. Проведенное исследование позволило выявить ряд особенностей, закономерностей и тенденций развития сложных социально-экономических систем и их влияния на устойчивое развитие региона, что может служить основой для работы над актуальными научно-практическими проблемами реализации инновационной деятельности на региональном уровне.

Ключевые слова: инновации; межорганизационное взаимодействие; сложные социально-экономические системы; устойчивое развитие региона

\section{ANALYSIS OF THEORETICAL APPROACHES AND INTERACTION MANAGEMENT PRACTICES WITHIN MAIN ENTITIES TO ENSURE SUSTAINABLE REGION DEVELOPMENT IN A STRATEGIC PERSPECTIVE}

\section{Kirillova E.A.}

In the existing concepts of competitive advantages sources determining tools for analyzing and evaluating resources, abilities and competencies in relation to cooperative formation are rather poorly presented. Although, as foreign practice shows, it is precisely such forms of joint organization of activities that currently have the greatest effectiveness. These features determined the purpose of this study - the analysis of existing theoretical 
approaches and proven practices for identifying and evaluating unique resources and abilities of complex socio-economic systems.

Method or methodology of the work: mainly general logical methods of analysis and synthesis, generalization were used. The results show that one of competitive advantages sources of modern organizations is the network of their partnerships, in which scientific organizations play an important role. At the same time, they are not static, but represent a constantly changing combination of properties, parameters, skills, competencies, interests and abilities, which determines the need to describe such competitive advantages through «action» abilities - the ability of the system to develop, learn, increase the volume of resources and increase the efficiency of their use through integration, formation, recombination and reconfiguration of key competencies in the interests of compliance with the changing external environment, thereby ensuring the strategic flexibility of the organization through the implementation of the maximum possible synergetic effect from the totality of available resources and competencies. There is an increasing need for such a model of innovation implementation as the "triple helix». Such a change in the composition of participants leads to a change in the movements of information, financial, material and personnel flows within the system of cooperative formation, transformation of its structure, targets and allocation of resources to achieve them. The roles of the subjects of interaction themselves are also changing quite a lot now. The conducted research made it possible to identify a number of features, patterns and trends in the development of complex socio-economic systems and their impact on the sustainable development of the region, which can serve as a basis for working on current scientific and practical problems of implementing innovative activities at the regional level.

Keywords: innovations; inter-organizational interaction; complex socio-economic systems; sustainable development of the region

В современных условиях в связи с усиливающимися тенденциями глобализации, развития сетевых форм организации и коммуникации все большее распространение получают процессы коопера- 
ционного взаимодействия и совместной реализации инновационных проектов. Принятие управленческих решений, используя ограниченные ресурсы, специализацию в рамках лишь одной из стадий инновационного процесса, обуславливают возросшую потребность в такой модели реализации инновационной деятельности как «тройная спираль», предусматривающей сотрудничество не только в сфере государственного управления, производства, но и в сфере научноисследовательских и опытно-конструкторских работ, направленное на их взаимное совершенствование.

Однако при этом вопросы управления развитием таких сложных социально экономических систем, как одного из важных факторов инновационного развития региона, остаются описанными достаточно фрагментарно, в том числе в связи с объективными сложностями оценки и анализа уникальных ресурсов, компетенций и динамических способностей субъектов «тройной спирали», обусловленные отсутствием трактовки данных категорий на уровне социальноэкономических образований, наличием значительного числа качественных параметров и высоким уровнем субъективизма при их расчете. Это определило актуальность исследования теоретических подходов и практик управления взаимодействием в рамках сложных социально-экономических систем для обеспечения устойчивого сбалансированного развития региона в стратегической перспективе, а также потенциала роста синергетических эффектов от динамического развития способностей таких объединений.

Стратегический потенциал современного предприятия описывается как совокупность уникальных ресурсов и способностей, вписанных в его организационную структуру, которые могут приобретаться в том числе в процессе обучения через действия [11]. Именно они лежат в основе стратегий кооперации между организациями в текущих быстроменяющихся условиях и определяют потенциал такого формирования. Стремительность изменений среды и слабая предсказуемость этих изменений стали толчком к смене фокуса в определении конкурентных преимуществ предприятия - от его позиции во внешней среде к наличию уникальных ресурсов и способ- 
ностей внутри самого предприятия. Эти факторы, согласно ресурсному подходу, определяют конкурентоспособность и устойчивость развития современной организации в стратегической перспективе. Развитием данного подхода стала концепция динамических способностей, направленная на преодоление ограничений в статичности ресурсного подхода и, стремящаяся учитывать помимо наличия совокупности уникальных ресурсов и компетенций, также способность системы развиваться, наращивать объемы этих ресурсов и повышать эффективность их использования [9]. Для оценки динамики изменений потенциала часто используются темпы прироста. Распространение данной концепции также связано с усилением роли нематериальных ресурсов и информационно-коммуникационных технологий, трансформацией теорий инновационного развития, становлением экономики знаний и влиянием на принятие решений практики неформального взаимодействия между стейкхолдерами, а также ростом сетевых форм организации бизнес-процессов. Изменения указанных факторов ставят перед субъектами экономических отношений задачу необходимости поиска и трансформации динамических способностей, обладающих возможностями реконфигурации ресурсов, процессов, отношений. Под «динамическими способностями» понимаются способности и навыки организации, которые имеют потенциал интегрирования, формирования и реконфигурации ключевых компетенций в интересах соответствующих изменений во внешней среде, тем самым обеспечивая стратегическую гибкость организации посредством реализации максимально возможного синергетического эффекта от совокупности имеющихся ресурсов и компетенций [6].

Несмотря на сложность идентификации и описания данной категории, наличия нескольких вариантов его понимания, вопрос оценки и представления динамических способностей организации в некоторой измеримой форме неоднократно рассматривался в работах российских и зарубежных ученых. Можно выделить применение имитационного моделирования для описания связи между результативностью организаций одной отрасли и их динамическими спо- 
собностями [24], методы сценарного анализа [1]. В [4] представлена методика построения трехкомпонентного вектора, отражающего статус и проблемность компетенции, а также интегральную оценку ее соответствия требованиям, предъявляемым к ключевым компетенциям. Расчет показателя «Сила динамических способностей предприятия», связанного со скоростью, с которой трудно имитируемые ресурсы предприятия могут быть трансформированы, при соответствии направления трансформации вектору изменений среды описан в [7].

В тоже время на уровне групп организаций, имеющих разный целевой характер деятельности и функционирующих совместно в рамках кооперационной сети, методических исследований не так много. Особенности таких сетевых формирований и необходимость учета специфики их создания, закономерностей развития, не позволяют использовать существующий для единичных субъектов инструментарий. Кроме того, в них не затрагиваются вопросы актуальные для таких сложных социально-экономических систем как: выбор участников взаимодействия, определение формы его реализации, интенсивность и направление развития, идентификация группы субъектов как единого формирования, значимость факторов пространственной и временной удаленности. В свою очередь инструменты и механизмы решения данных задач, применительно к субъектам одного или близкого направления деятельности, но находящихся на разных этапах цепочки создания стоимости нашли отражение в [8,9]. Такое внимание к сетевым формам организации деятельности со стороны промышленных предприятий и организаций сферы услуг обусловлено желаниями сокращения издержек и получения прибыли от эффекта масштаба. В [10] акцент сделан на оценку системного эффекта от взаимодействия промышленных предприятий и описана методика его оценки на основе показателей системной сочетаемости. Достаточно близкая категория кооперационного левереджа, рассчитываемого как произведение степени (эластичности) влияния интенсивности промышленной кооперации на эффективность системы и статистической силы этого влия- 
ния, измеряемой коэффициентом детерминации представлена в [2]. Описанная методика направлена на оценку мультиплицирующего влияния интенсивности промышленной кооперации социальноэкономической системы на эффективность ее функционирования. Деятельность многих из рассматриваемых в данных работах организаций носит международный характер, где подтверждается особая значимость совместной деятельности, так как она дает участникам преимущества от размещения производства в перспективных регионах, преодоление барьеров при выходе на новые рынки и др. [11]. Выбор партнера взаимодействия имеет важное значение для успеха такого стратегического альянса [17].

В тоже время все больше современных исследований подчеркивают необходимость расширения состава взаимодействующих субъектов для создания стратегических инноваций, способных обеспечивать устойчивое развитие субъектов в длительной перспективе [19]. Взаимодействие субъектов в рамках инновационной деятельности выходит за рамки традиционной линейной модели, поскольку сотрудничество может не ограничиваться совместными исследованиями и охватывать различного рода наукоемкие услуги, развитие человеческого капитала или усиление внутренних компетенций. Наличие разветвленной партнерской сети служит явным признаком высокого инновационного потенциала предприятия. Подобное взаимодействие опирается на культурную общность, позволяющую преодолевать барьеры кооперации, связанные с различиями в стратегиях управления, «целевых функциях» предприятий реального сектора экономики и научных организаций [19]. Обуславливается возросшая потребность в такой модели реализации инновационной деятельности как «тройная спираль» [15], предусматривающей сотрудничество не только в сфере государственного управления, производства, но и в сфере научно-исследовательских и опытно-конструкторских работ, направленное на их взаимное совершенствование. Среди российских предприятий, вовлеченных в такое взаимодействие, это пока достаточно небольшое число субъектов, но они выделяются как обладающие способностью к формированию ключевых элементов стратегиче- 
ского потенциала и устойчивыми конкурентными преимуществами не только на уровне самих субъектов, но и группы. Как отмечается в [19] такие предприятия сравнительно чаще выступают проактивными операторами технологических рынков, вовлекаясь в процессы создания и распространения нематериальных активов, в том числе будучи частью процессов регистрации объектов интеллектуальной собственности и трансфером прав на них. Такое изменение состава участников приводит к изменению движений информационных, финансовых и материальных потоков внутри системы кооперационного формирования, трансформации ее структуры, целевых установок и распределения ресурсов для их достижения. Сложность построения такой системы также связана с разнонаправленностью стратегий деятельности предприятий реального сектора экономики и науки, вызванной как институциональной и корпоративной спецификой, так и их структурными особенностями [19]. Со стороны научно-исследовательский организаций все еще достаточно распространена традиционная модель «Североамериканской школы», которая характеризуется поступательным увеличением сложности методов исследования, что делает их еще более фундаментальным и отдаленным от заинтересованных субъектов реальных бизнес-систем. С другой стороны, на выбор темы научных исследований также влияют доступность и академические нормы. Исследования проводятся по темам с использованием методов, при которых данные легче собирать. Такие стратегии не способствует созданию устойчивой основы для изучения процессов и инструментов актуальных и востребованных рынком. Кроме того, сотрудничество между научными организациями и предприятиями реального сектора экономики зависит от типа и структуры процессов обмена знаниями, в которых участвуют эти организации. Также имеют важное значение размер и вид деятельности предприятий. Так, организации с государственным участием имеют выраженную склонность устанавливать связи с другими институциональными партнерами [19].

Роли самих субъектов взаимодействия сейчас также достаточно сильно меняются. Традиционно основной задачей университетов 
считалось формирование человеческого капитала и развитии науки. Однако в последние десятилетия акцент сместился в сторону их инновационной функции. Университеты становятся все более активными в организации взаимодействия с промышленными предприятиями, стремясь не только создавать объекты интеллектуальную собственность, но и участвовать в процессах их внедрения и трансфера технологий. Они, согласно концепции «треугольник знаний», становятся идеальными источниками инновационный идей, предложений и предпринимательских практик при организации эффективной системы двунаправленного циркулирования между ними потоков знаний. Для возникновения таких потоков требуются специальные инструменты, позволяющие мобилизовать необходимые ресурсы для создания инновационных продуктов и услуг, из которых участники «сети знаний» (стейкхолдеры), включая представителей общественности, частного бизнеса и академического сектора, смогут извлечь преимущества [20]. Их отсутствие в существующих сейчас системах препятствует использованию университетских знаний в качестве конкурентного актива. Отмечается также потребность вовлечения предпринимательских университетов в их новой роли в деятельность регионов, в которых расположены эти учреждения. Исследователям необходимо опираться на экономические и социальные условия территории их локализации [18]. Кроме того, продолжительность кооперации обусловливается уровнем доверия, глубиной взаимодействия между участниками, степенью их «институциональной близости» [19]. Изменение состава участников кооперационного взаимодействия в связи с уже отмеченными особенностями скорее всего потребует преодолеть значительную «дистанцию» [20].

Можно отметить ряд исследований рассматривающих межорганизационые связи и сети с позиции теорий пространственного размещения и развития территорий, где в качестве целевых и оценочных показателей акцент делается на их влиянии на изменения конкурентоспособности региона локализации этой сети. Так, вопросы географического размещения элементов в цепочках создания 
стоимости носят решающий характер при обосновании кластерной политики и «умной специализации» [12]. Создание и развитие системы эффективных межорганизационных связей, концентрации усилий отдельных субъектов, как основы конкурентоспособности территорий и предпосылки к устойчивости экономического роста отмечается в работах $[3,5]$. Кроме того географический фактор можно рассматривать с одной стороны как степень пространственной удаленности субъектов и, следовательно, сложность и уровень издержек по организации такого взаимодействия, а с другой как основу исторической культурно-экономической общности партнеров по кооперации - общее понимание контекста взаимодействия, схожий набор ресурсов и доступ к ним, близкая система ценностей и требований, в том числе по обмену информацией и другими ресурсами [19], что играет существенную роль.

Достаточно много внимания при описании сетей и инструментов их управления уделяется балансу и координации интересов отдельных участников, а также возможностям и ограничениям, которые они накладывают на процесс создания ценностей [23]. В первоначальных концепциях власти и влияния реляционное взаимодействие было определено как непрерывный процесс балансирования взаимных состояний власти и зависимости $[13,14]$, сначала применимо к отношениям субъектом бизнес среды, а затем и международным сетям [21]. Близость расстояния между партнерами по кооперации и, следовательно, эффективность их отношений сильно зависит от постоянного баланса доверия, зависимости и власти [22]. Кооперационные формы совместной работы сокращают и/или изменяют характер «дистанции» между академическими исследователями и бизнесом [16]. Достаточность точек соприкосновения между учеными и практиками определяется их стремлением к общим целям, необходимым для совместного создания знаний.

Таким образом, исследование показало, что стратегический потенциал современной организации характеризуется совокупностью уникальных ресурсов и способностей, имеющихся внутри самой организации и сети ее партнерств, важную роль в которой играют 
научные организации. Именно эти факторы определяют ее конкурентоспособность и устойчивость развития в стратегической перспективе. Но они не статичны, а представляют собой постоянно меняющуюся комбинацию свойств, параметров, навыков, компетенций, интересов и способностей, что определяет потребность в описании таких конкурентных преимуществ через способности «действия» - способность системы развиваться, обучаться, наращивать объемы ресурсов и повышать эффективность их использования за счет интегрирования, формирования, рекомбинации и реконфигурации ключевых компетенций, в интересах соответствия изменяющейся внешней среды, тем самым обеспечивая стратегическую гибкость организации посредством реализации максимально возможного синергетического эффекта от совокупности имеющихся ресурсов и компетенций. Несмотря на сложность идентификации и описания данной категории, наличия нескольких вариантов его понимания вопрос оценки и представления динамических способностей организаций в некоторой измеримой форме неоднократно рассматривался в работах российских и зарубежных ученых. В тоже время на уровне групп субъектов, имеющих разный целевой характер деятельности и функционирующих совместно в рамках кооперационной сети, методических исследований не так много. Особенности таких сетевых формирований и необходимость учета специфики их создания, закономерностей развития, не позволяют использовать существующий для единичных субъектов инструментарий.

В тоже время все больше современных исследований подчеркивают необходимость расширения состава взаимодействующих субъектов для создания стратегических инноваций, способных обеспечивать устойчивое развитие субъектов в длительной перспективе. Возрастает потребность в такой модели реализации инновационной деятельности как «тройная спираль». Такое изменение состава участников приводит к изменению движений информационных, финансовых и материальных потоков внутри системы кооперационного формирования, трансформации ее структуры, целевых установок и распределения ресурсов для их достижения. Среди 
российских предприятий, вовлеченных в такое взаимодействие, это пока достаточно небольшое число субъектов, что обусловлено рядом трудностей.

Роли самих субъектов взаимодействия сейчас также достаточно сильно меняются. Университеты становятся все более активными в сотрудничестве с промышленными предприятиями, стремясь не только создавать объекты интеллектуальную собственность, но и содействовать передаче технологий. Они, согласно концепции «треугольник знаний», становятся идеальными источниками инновационный идей, предложений и предпринимательских практик при организации эффективной системы двунаправленного циркулирования между ними потоков знаний. Но отсутствие специальные инструменты, позволяющие мобилизовать необходимые ресурсы для создания инновационных продуктов и услуг, в свою очередь, может препятствовать использованию университетских знаний в качестве конкурентного актива.

Географический фактор можно рассматривать с одной стороны как степень пространственной удаленности субъектов и, следовательно, сложность и уровень издержек по организации такого взаимодействия, а с другой - как основу исторической культурноэкономической общности партнеров по кооперации -общее понимание контекста взаимодействия, что играет существенную роль. Достаточно много внимания при описании сетей и инструментов их управления уделяется балансу и координации интересов отдельных участников, а также возможностям и ограничениям, которые они накладывают на процесс создания ценностей.

Проведенное исследование теоретических подходов и практик управления взаимодействием в рамках сложных социально-экономических систем для обеспечения устойчивого сбалансированного развития региона в стратегической перспективе позволило выявить ряд особенностей и тенденций развития данной области, что может служить основой для работы над такими актуальными проблемами, как количественная оценка вклада научно-исследовательской деятельности, результатов интеллектуальной деятельности и 
устойчивого роста; определение типов потенциальных участников взаимодействия для наращения инновационного потенциала; сравнение стратегий предприятий в области инноваций и результатов их деятельности.

Работа выполнена при финансовой поддержке "Совета по грантам Президента Российской Федераџии для государственной поддержкки молодых российских ученых - кандидатов наук» по проекту MK-4087.2021.2.

\section{Список литературы}

1. Бек Н.Н., Сарычев А.Е. Динамический стратегический анализ: ориентация на устойчивость конкурентного преимущества компании в условиях динамизма и глобализации рынков // Модернизация экономики и глобализация. В 3 книгах (отв. ред. Е. Г. Ясин). М.: ИДГУВШЭ, 2009. Т. 3. С. 97-106.

2. Булатов А.Н. Методология стратегического управления промышленной кооперацией: дис. ... д-р. экон. наук. Казань, 2011. 451 с.

3. Васильева 3.А., Бородкина В.В. Новые тенденции в развитии межорганизационных связей и их влияние на качество и устойчивость экономического роста // Общество: политика, экономика, право. 2016. № 11. С. 29-34.

4. Гилева Т.А. Компетенции организации: инструменты анализа и оценки // Вестник Пермского национального исследовательского политехнического университета. Социально-экономические науки. 2018. № 3. С. 266-276. DOI: https://doi.org/10.15593/22249354/2018.3.21

5. Заенчковский А.Э. Методология логистического и организационного управления инновационной средой региональных научно-промышленных комплексов // Российское предпринимательство. 2013. №14. C. 129-137.

6. Кулеш В.А. Концепция динамических способностей в стратегическом выборе компании выборе компании // Научный журнал КубГАУ. 2015. № 108(04), URL: http://ej.kubagro.ru/2015/04/pdf/86.pdf 
7. Никифорова Н.С. Управление промышленным предприятием по показателям его динамических способностей: дис. ... канд. экон. наук. Челябинск, 2020. 214 с.

8. Сабаткоев Т.Р. Развитие горизонтальной кооперации контрагентов цепей поставок предприятий пищевой отрасли: дис. ... канд. экон. наук. Москва, 2016. 134 с.

9. Старикова М.С. Теория и методология адаптивного управления промышленной корпорацией на основе эффективных деловых взаимодействий: дис. ... д-ра экон. наук. Белгород, 2015. 399 с.

10. Федосеев А.В. Исследование и оценка эффективности интеграции промышленных предприятий: дис. ... канд. экон. наук. Челябинск, 2009. $140 \mathrm{c}$.

11. Чуйкин A.M. Международная кооперационная способность менеджмента в стратегическом потенциале организации // Вестник Балтийского федерального университета им. И. Канта. Серия: Гуманитарные и общественные науки. 2010. № 9. С. 107-117.

12. Balland P.-A., Boschma R., Crespo J., Rigby D.L. Smart specialization policy in the European Union: Relatedness, knowledge complexity and regional diversification // Regional Studies, 2018, no. 35 (4), pp. 1-17.

13. Cook K.S., Emerson R.M., Gillmore M.R., Yamagishi T. The Distribution of Power in Exchange Networks: Theory and Experimental Results // The American Journal of Sociology, 1983, vol. 89, № 2, pp. 275-305.

14. Emerson R.M. Power-Dependence Relations // American Sociological Review, 1962, vol. 27, № 1, pp. 31-41.

15. Etzkowitz H, Leydesdorff L. The dynamics of innovation: from national systems and «Mode 2» to a triple helix of university-industry-government relations // Res Policy, 2000, №29(2), pp. 109-123.

16. Gulati Ranjay, Nitin Nohria, Akbar Zaheer. Strategic Networks // Strategic Management Journal, 2000, № 21 (3), pp. 203-15.

17. Kanungo R.P. Learning success factors of strategic alliances and estimating under an alternate specification // Investment Management and Financial Innovations, 2015, vol. 12, is. 3, pp. 120-131.

18. Mascarenhas C., Ferreira J.J., Marques C. University-industry cooperation: A systematic literature review and research agenda// Science 
and Public Policy, 2018, vol. 45, is. 5, pp. 708-718. DOI: https://doi. org $/ 10.1093 /$ scipol/scy003

19. Vlasova V., Roud V. Cooperative Strategies in the Age of Open Innovation: Choice of Partners, Geography and Duration // Foresight and STI Governance, 2020, vol. 14, no. 4, pp. 80-94. DOI: https://doi. org/10.17323/2500-2597.2020.4.80.94

20 . Vonortas N. The role of universities in the knowledge triangle // Foresight and STI Governance, 2017, vol. 11, no. 2, pp. 6-8. DOI: https:// doi.org/10.17323/2500-2597.2017.2.6.8

21. Wathne K.H., Heide J.B., Mooi E.A., Kumar A. Relationship governance dynamics: the roles of partner selection efforts and mutual investments // Journal of Marketing Research, 2018, vol. 55(5), pp. 704-721.

22. Yang J. Towards the restructuring and co-ordination mechanisms for the architecture of chinese transport logistics. Erasmus University Rotterdam, Erasmus Research Institute of Management, 2009. 184 p.

23. Young L., Freytag P.V. Beyond research method to research collaboration: Research co-production relationships with practitioners // Industrial Marketing Management, 2021, vol. 92, pp. 244-253. DOI: https://doi. org/10.1016/j.indmarman.2020.02.016

24.Zott C. Dynamic capabilities and the emergence of intraindustrial differential firm performance: insights from a simulation study // Strategic Management Journal, 2003, 24 (2), pp. 97-125.

\section{References}

1. Bek N.N., Sarychev A.E. Dinamicheskiy strategicheskiy analiz: orientatsiya na ustoychivost' konkurentnogo preimushchestva kompanii v usloviyakh dinamizma i globalizatsii rynkov [Dynamic strategic analysis: focusing on the sustainability of the company's competitive advantage in the context of dynamism and globalization of markets]. Modernizatsiya ekonomiki i globalizatsiya [Modernization of the Economy and Globalization]. M.: IDGU-VShE, 2009, vol. 3, pp. 97-106.

2. Bulatov A.N. Metodologiya strategicheskogo upravleniya promyshlennoy kooperatsiey [Methodology for strategic management of industrial cooperation]. Kazan, 2011, 451 p. 
3. Vasil'eva Z.A., Borodkina V.V. Novye tendentsii v razvitii mezhorganizatsionnykh svyazey i ikh vliyanie na kachestvo i ustoychivost' ekonomicheskogo rosta [The new trends in the development of intercorporate relations and their impact on the quality and sustainability of economic growth]. Obshchestvo: politika, ekonomika, pravo, 2016, № 11, pp. 29-34.

4. Gileva T.A. Kompetentsii organizatsii: instrumenty analiza i otsenki [Organization competencies: analysis and assessment tools]. Vestnik Permskogo natsional'nogo issledovatel'skogo politekhnicheskogo universiteta. Sotsial'no-ekonomicheskie nauki, 2018, no. 3, pp. 266-276. DOI: https://doi.org/10.15593/2224-9354/2018.3.21

5. Zaenchkovskiy A.E. Metodologiya logisticheskogo i organizatsionnogo upravleniya innovatsionnoy sredoy regional'nykh nauchno-promyshlennykh kompleksov [Methodology of logistic and organizational management of the innovative environment of regional scientific and industrial complexes]. Rossiyskoe predprinimatel'stvo, 2013, no. 14, pp. 129-137.

6. Kulesh V.A. Kontseptsiya dinamicheskikh sposobnostey v strategicheskom vybore kompanii vybore kompanii [The concept of dynamic abilities in the strategic choice of the company]. Nauchnyy zhurnal KubGAU, 2015, no. 108(04). http://ej.kubagro.ru/2015/04/pdf/86.pdf.

7. Nikiforova N.S. Upravlenie promyshlennym predpriyatiem po pokazatelyam ego dinamicheskikh sposobnostey [Industrial enterprise management in terms of dynamic capabilities]. Chelyabinsk, 2020, 214 p.

8. Sabatkoev T.R. Razvitie gorizontal'noy kooperatsii kontragentov tsepey postavok predpriyatiy pishchevoy otrasli [Development of horizontal cooperation of counterparties in the supply chains of food industry enterprises]. M., 2016, 134 p.

9. Starikova M.S. Teoriya i metodologiya adaptivnogo upravleniya promyshlennoy korporatsiey na osnove effektivnykh delovykh vzaimodeystviy [Theory and methodology of adaptive management of an industrial corporation based on effective business interactions]. Belgorod, 2015, $399 \mathrm{p}$.

10. Fedoseev A.V. Issledovanie i otsenka effektivnosti integratsii promyshlennykh predpriyatiy [Research and evaluation of the effectiveness of the integration of industrial enterprises]. Chelyabinsk, 2009, 140 p. 
11. Chuykin A.M. Mezhdunarodnaya kooperatsionnaya sposobnost' menedzhmenta $\mathrm{v}$ strategicheskom potentsiale organizatsii [International cooperation ability of management in the strategic potential of the organization]. Vestnik Baltiyskogo federal'nogo universiteta im. I. Kanta. Seriya: Gumanitarnye i obshchestvennye nauki. 2010, no. 9, pp. 107-117.

12.Balland P.-A., Boschma R., Crespo J., Rigby D.L. Smart specialization policy in the European Union: Relatedness, knowledge complexity and regional diversification. Regional Studies, 2018, no. 35 (4), pp. 1-17.

13. Cook K.S., Emerson R.M., Gillmore M.R., Yamagishi T. The Distribution of Power in Exchange Networks: Theory and Experimental Results. The American Journal of Sociology, 1983, vol. 89, no. 2, pp. 275-305.

14. Emerson R.M. Power-Dependence Relations. American Sociological Review, 1962, vol. 27, no. 1, pp. 31-41.

15. Etzkowitz H, Leydesdorff L. The dynamics of innovation: from national systems and «Mode 2» to a triple helix of university-industry-government relations. Res Policy, 2000, no. 29(2), pp. 109-123.

16. Gulati Ranjay, Nitin Nohria, Akbar Zaheer. Strategic Networks. Strategic Management Journal, 2000, no. 21 (3), pp. 203-15.

17. Kanungo R.P. Learning success factors of strategic alliances and estimating under an alternate specification. Investment Management and Financial Innovations, 2015, vol. 12, is. 3, pp. 120-131.

18. Mascarenhas C., Ferreira J.J., Marques C. University-industry cooperation: A systematic literature review and research agenda. Science and Public Policy, 2018, vol. 45, is. 5, pp. 708-718. DOI: https://doi.org/10.1093/scipol/scy003

19. Vlasova V., Roud V. Cooperative Strategies in the Age of Open Innovation: Choice of Partners, Geography and Duration. Foresight and STI Governance, 2020, vol. 14, no. 4, pp. 80-94. DOI: https://doi. org/10.17323/2500-2597.2020.4.80.94

20 . Vonortas N. The role of universities in the knowledge triangle. Foresight and STI Governance, 2017, vol. 11, no. 2, pp. 6-8. DOI: https://doi. org/10.17323/2500-2597.2017.2.6.8

21. Wathne K.H., Heide J.B., Mooi E.A., Kumar A. Relationship governance dynamics: the roles of partner selection efforts and mutual investments. Journal of Marketing Research, 2018, vol. 55(5), pp. 704-721. 
22. Yang J. Towards the restructuring and co-ordination mechanisms for the architecture of chinese transport logistics. Erasmus University Rotterdam, Erasmus Research Institute of Management, 2009, 184 p.

23. Young L., Freytag P.V. Beyond research method to research collaboration: Research co-production relationships with practitioners. Industrial Marketing Management, 2021, vol. 92, pp. 244-253. DOI: https://doi. org/10.1016/j.indmarman.2020.02.016

24.Zott C. Dynamic capabilities and the emergence of intraindustrial differential firm performance: insights from a simulation study. Strategic Management Journal, 2003, no. 24 (2), pp. 97-125.

\section{ДАННЫЕ ОБ АВТОРЕ}

Кириллова Елена Александровна, доцент кафедры «Информационные технологий в экономике и управлении», кандидат экономических наук, доцент филиал ФГБОУ ВО «НИУ «МЭИ» в г. Смоленске пр. Энергетический, 1, г. Смоленск, Смоленская область, 214013, Российская Федерачия kirillova.el.al@yandex.ru

\section{DATA ABOUT THE AUTHOR}

Elena A. Kirillova, Associate Professor «Accounting, Analysis and Audit», Candidate of Economic Sciences, Associate Professor Branch of the National Research University "Moscow Power Engineering Institute» in Smolensk

1, Energy passage, Smolensk, Smolensk region, 214013, Russian Federation

kirillova.el.al@yandex.ru

SPIN-code: 8543-9450

ORCID: 0000-0001-7046-3316

Scopus Author ID: 57210751002

Поступила 23.11.2021

Received 23.11.2021

После рецензирования 26.11.2021

Revised 26.11.2021

Принята 29.11.2021

Accepted 29.11.2021 